Charts for assisting in the Selection of Suitable Glasses for Cemented Doublets

This content has been downloaded from IOPscience. Please scroll down to see the full text. 1918 Trans. Opt. Soc. 19169

(http://iopscience.iop.org/1475-4878/19/6/302)

View the table of contents for this issue, or go to the journal homepage for more

Download details:

IP Address: 129.78.139.28

This content was downloaded on 02/10/2015 at 00:47

Please note that terms and conditions apply. 


\title{
Charts for assisting in the Selection of Suitable Glasses for Cemented Doublets.
}

\author{
By T. SMITH, B.A.
}

(From the National Physical Laboratory.)

A METHOD of using two charts as an aid in the selection of pairs of glasses for cemented objectives free from spherical and chromatic aberrations and coma when used at a given magnification has been described by the author in a paper read before the Physical Society.* The charts shown here are used in a similar way, but instead of being constructed to give an exact solution to a definite problem, they are intended to serve as a guide to a manufacturer or computer in selecting a pair of glasses for any purpose. As this appears to be a rather wide claim to make, a short explanation may be given of the facts which justify it.

Objectives of the type considered can be calculated from expressions for the first order aberrations when thicknesses are neglected. The aberrations of such thin lenses are determined by three quantities which depend only on the relative powers of the two component lenses and on their refractive indices. One of these quantities is the Petzval sum, denoted by $\tilde{\omega}$, and this does not change when the form of the lens is altered by adding a common increment of curvature to each refracting surface. The other two quantities are denoted by $C_{0}$ and $\left(B_{0}^{\prime}-B_{0}\right)$, which become $C$ and $\left(B^{\prime}-B\right)$ if the curvature of each surface is increased by some value, say $r$, when the focal length is unity. The relations between these quantities are

$$
\begin{gathered}
\mathrm{C}=\mathrm{C}_{0}+r^{2}(1+2 \tilde{\omega}) \\
\mathrm{B}^{\prime}-\mathrm{B}=\mathrm{B}_{0}^{\prime}-\mathrm{B}_{0}+2 r(1+\widetilde{\omega})
\end{gathered}
$$

These three quantities $\tilde{\omega},\left(B_{0}^{\prime}-B_{0}\right)$, and $C_{0}$ thus determine the aberrations of every thin lens made from the same two glasses which have the same type of colour correction. Now $\widetilde{\omega}$ always lies within small limits, and $\mathrm{B}_{0}^{\prime}-\mathrm{B}_{0}$ is always small. On the other hand, $\mathrm{C}_{0}$ varies over a wide range of values, and it is the variation in these values which gives the different character to the kinds of correction that are obtainable in different combinations of two glasses. In selecting glasses an average value of $\widetilde{\omega}$ may be assumed, and zero substituted for $\left(B_{0}^{\prime}-B_{0}\right)$ to a first approximation. All glass pairs which have the same value for $C_{0}$ when the required colour condition is satisfied can then be

* "The Choice of Glass for Cemented Objectives." Proc. Phys. Soc. 28 (1916), 220. 


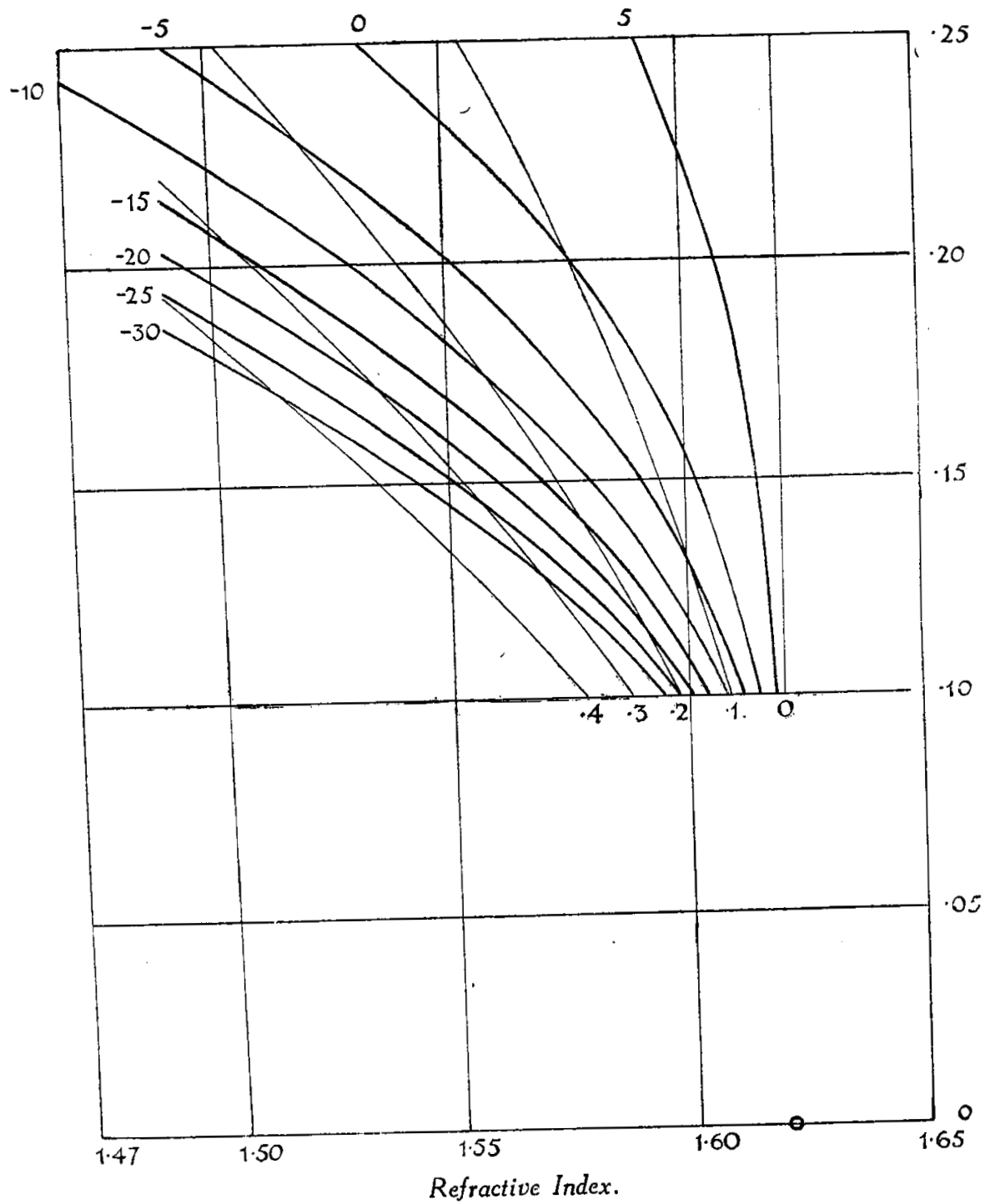

Fig. 1. - Chart for Spherical Aberrations. 


\section{Charts for Selection of Glasses for Cemented Doublets. 171}

substituted for one another and will enable the same types of spherical correction to be obtained within close limits.

In practice it is more convenient to take $4 \mathrm{C}_{0}+2 \tilde{\sigma}+1$ as the variable instead of $\mathrm{C}_{0}$, because $\mathrm{C}_{0}$ always occurs in this form in the expressions for the first order aberrations of thin lenses. The conditions which have most usually to be considered in lenses of this type are those relating to spherical aberration and to coma. The first order latitudinal displacements due to these aberrations are proportional to

and

$$
(1-m)^{4}\left\{4 \mathrm{C}+2 \tilde{\omega}+1-4\left(\mathrm{~B}^{\prime}-\mathrm{B}\right) \mathrm{M}+(3+2 \tilde{\omega}) \mathrm{M}^{2}\right\}
$$

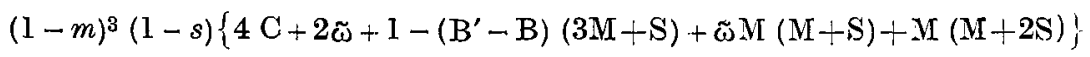

respectively where $m$ is the linear magnification of the object produced by paraxial rays, $s$ is the linear magnification of the stop similarly determined, and

$$
\mathrm{M}=\frac{1+m}{1-m} \quad, \quad \mathrm{~S}=\frac{1+s}{1-s}
$$

Thus if freedom from both aberrations is desired for $m=-1$, the conditions to be satisfied are

$$
4 \mathrm{C}+2 \tilde{\omega}+1=\mathrm{B}^{\prime}-\mathrm{B}=0
$$

Since $\mathrm{B}_{0}^{\prime}-\mathrm{B}_{0}$ is small, $r$ will be small, and therefore $\mathrm{C}$ will be very nearly equal to $C_{0}$. Glasses may, therefore, be selected by imposing the condition

$$
4 \mathrm{C}_{0}+2 \tilde{\omega}+1=0 \text {. }
$$

If more than one pair can be found which satisfy this condition equally well and there are no very obvious reasons for selecting one pair in preference to another (on such grounds, for example, as a greater difference in the $v$ values of the two glasses, leading to shallower surfaces), the selection may be determined by comparing the corrected conditions in which the actual values of $\tilde{\omega}$ and $B_{0}^{\prime}-B_{0}$ for the various pairs selected are substituted for the average value of $\tilde{\omega}$ and for zero respectively.

Advantage is taken of this property of $C_{0}$ or of $4 C_{0}+2 \tilde{\omega}+1$ in determining the types of correction that can be obtained by drawing curves connecting glasses having common values for this quantity. Since there are three variables - two refractive indices and the ratio of two powers - one of these must be fixed to enable a two dimensional diagram to be obtained. It is convenient to take the refractive index of one of the glasses as the fixed quantity. Since many lens combinations of widely differing character include a dense flint glass of refractive index about 1.62 , one very useful chart has this as the fixed quantity. Such a chart is shown in Fig. 1. The refractive index of the other 
glass is taken as abscissa, and the ordinate is the logarithm of the numerical value of the fraction

$$
\frac{\text { power of lens of variable refractive index }}{\text { power of lens of fixed refractive index }}
$$

The position of the fixed glass is represented in Fig. 1 by a circle near the lower right corner, and the thick curves correspond to glasses which

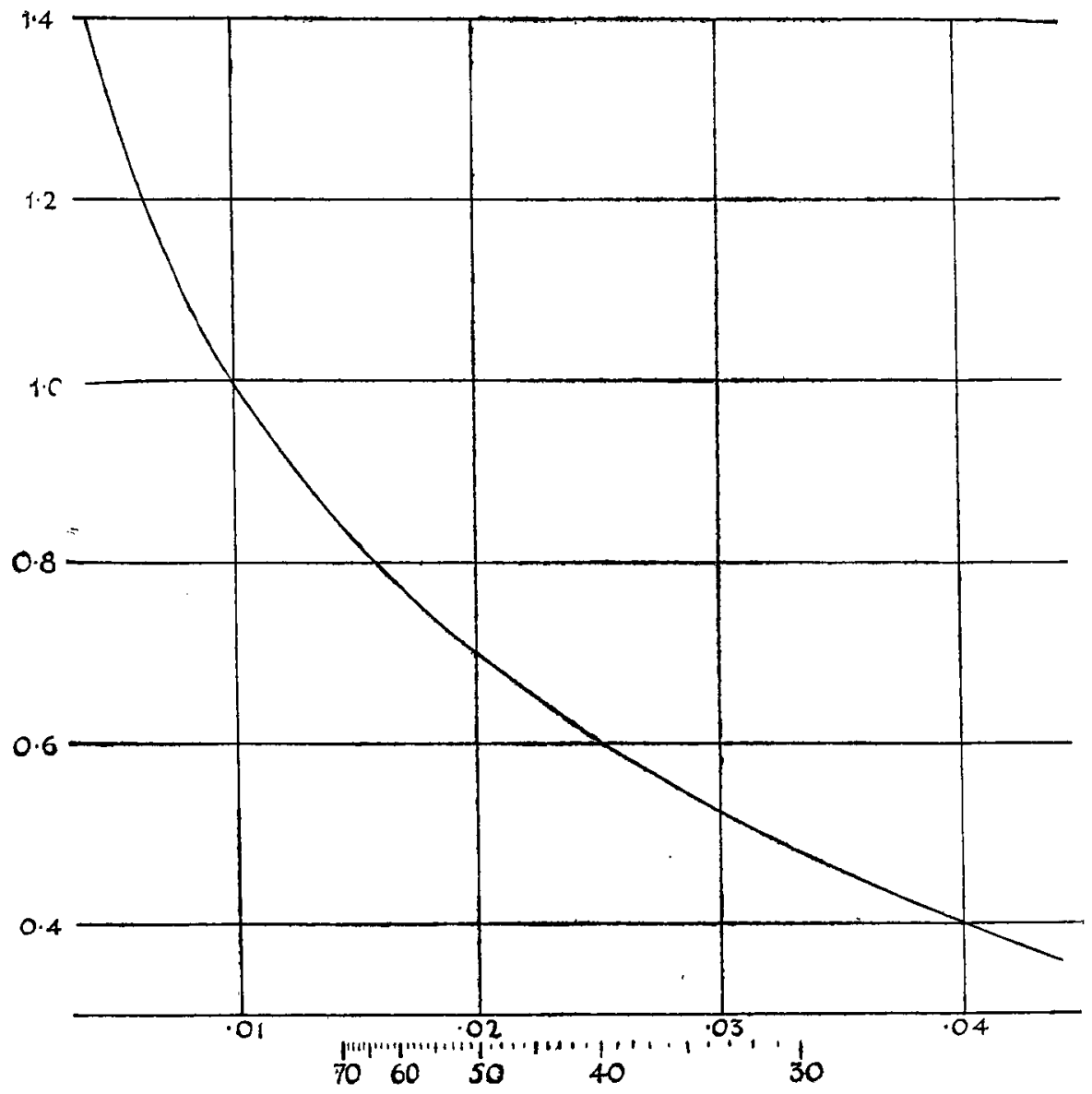

Fig. 2.-Chart for making Chromatic Allowances in constructing glass DIAGRAMS. 
in combination with the fixed glass give values of $4 \mathrm{C}_{0}+2 \tilde{\omega}+1$ ranging from +5 to -30 in steps of 5 . The thin curves give the values of $\left(\mathrm{B}_{0}^{\prime}-\mathrm{B}_{0}\right)$ in steps of $0^{\circ} 1$ from $0^{\circ} 0$ to $0^{\circ} 4$. The curves are not continued for values of the logarithm below $0^{\prime} 1$, as this region is of no practical interest.

Fig. 1 is used in conjunction with a second chart, such as Fig. 3, which is constructed from lists of the kinds of glass that are available. The chart shown in Fig. 3 shows glasses taken from a recent list of Messrs. Chance Bros. As before, the refractive index of the glass is taken as abscissa, and the logarithm of the $\nu$ as the ordinate. Classes shown in the catalogue in thick type as standard varieties which can be supplied from stock are indicated by two concentric circles, and other kinds by a single circle. It is convenient to write by each circle the catalogue number of the glass to which it refers, but this has not been done in this diagram as the numbers may not print satisfactorily on the small scale to which the originals must be reduced. One of these diagrams - say the glass chart-must be drawn on tracing paper. This is then laid over the chart reproduced in Fig. 1, both having been drawn to the same scales, with the lines corresponding to the same refractive indices superposed. The second diagram may then be moved in the direction in which the logarithms are measured through any distance, so as to bring the reference circle of Fig. $I$ and the circle representing any glass of refractive index 1.62 into coincidence. The other glass to be used to secure a given value of $4 \mathrm{C}_{0}+2 \widetilde{\omega}+1$ in a cemented achromatic objective is then found by choosing the one whose representative circle most nearly falls on the line of Fig. 1 corresponding to this required value. The theory of this procedure is plain, and depends upon the relation

$$
\log y-\log v^{\prime}=\log v / v^{\prime} .
$$

From the use of the two charts described it is then evident that the relation $\nu / v^{\prime}=-K / K^{\prime}$ will be satisfied, where $K$ and $K^{\prime}$ are the powers of the component lenses. The compound lens will thus be achromatic. It is obviously essential that charts to be used in this way should have as ordinates the logarithms of the fundamental variables, rather than the values of the variables themselves.

If this were the only use that could be made of the charts there would be very little justification for the labour of constructing them. Two facts, however, render it possible to use them in a much more general way. The shapes of the curves shown in Fig. 1 do not vary rapidly as the fundamental refractive index is changed, so that the one chart may be used for any fundamental refractive index which does not differ very considerably from 1'62. Three charts, for instance, should prove 


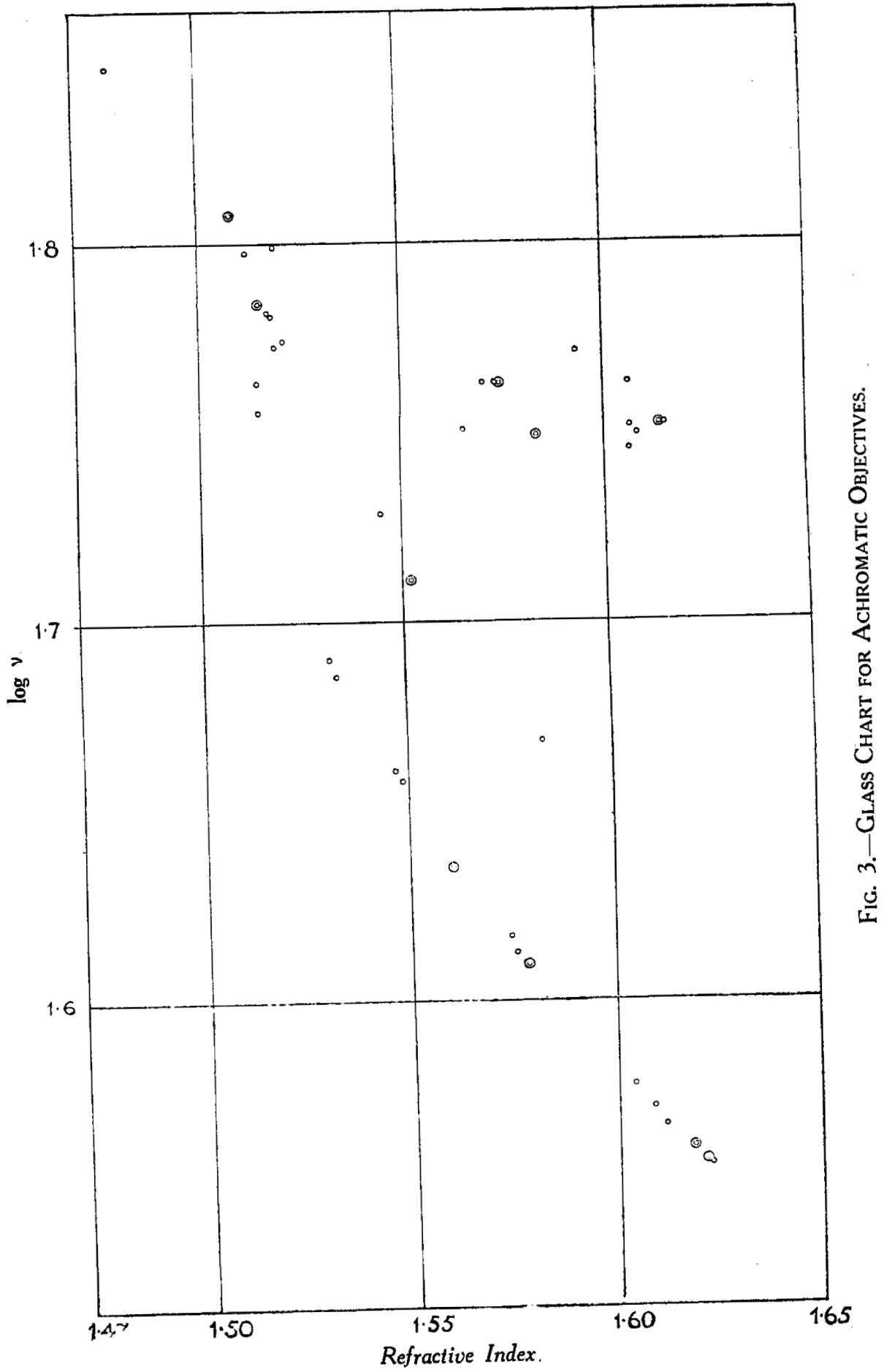




\section{Charts for Selection of Glasses for Cemented Doublets. 175}

ample for the whole range of refractive indices available for ordinary purposes. This means that the glass chart may be given a displacement in the direction in which the refractive index is measured relative to that on which it is placed, so that instead of requiring the straight lines corresponding to a common index to be superposed it is sufficient that

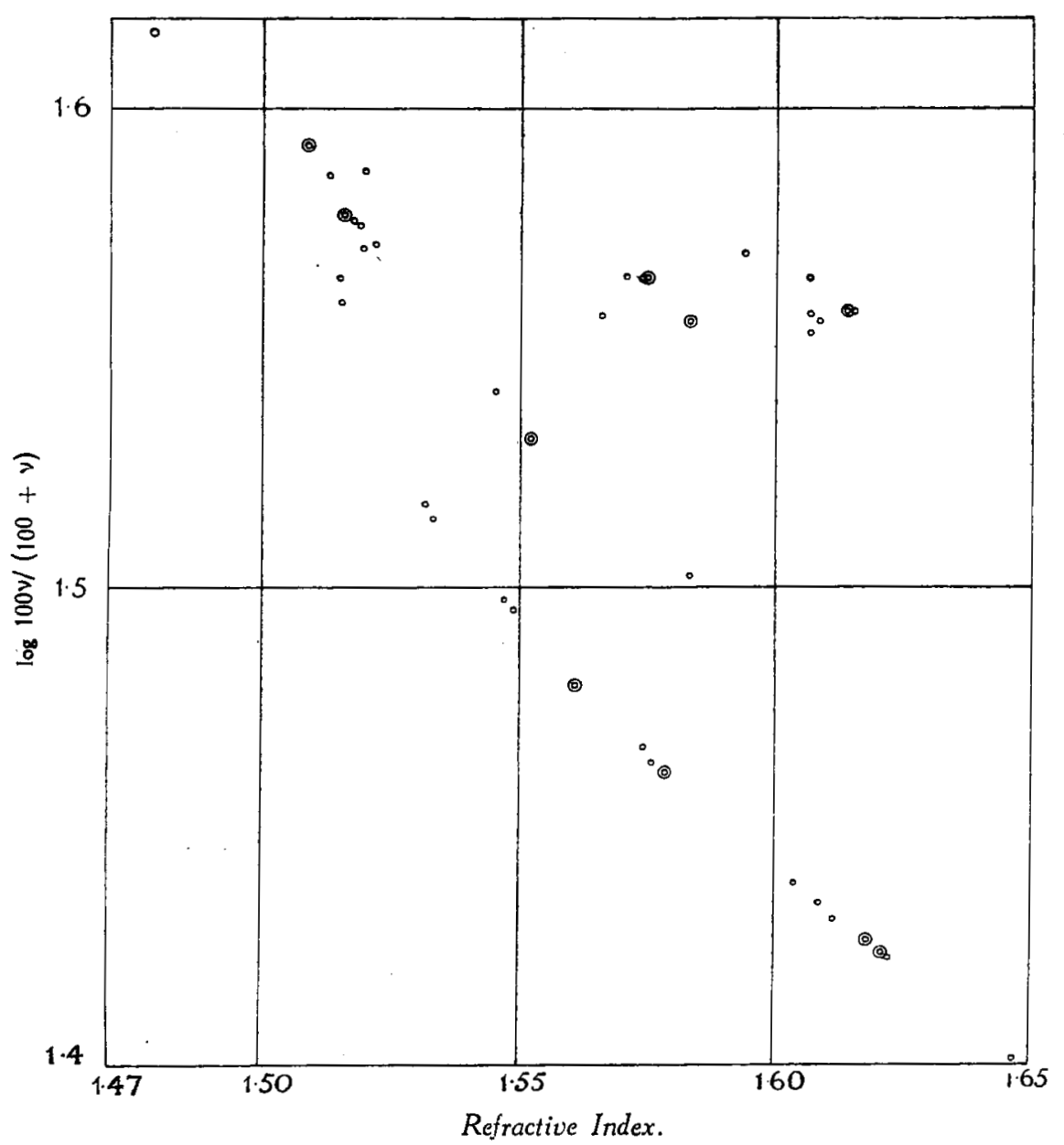

Fic. 4.-Glass Chart for Overcorrected Chromatic Aberration. 
they should be parallel to one another. Fig. 1, for example, may be used for any glass combination in which any dense flint is used.

The second consideration is that by substituting for Fig. 3 another diagram in which the ordinates are changed, any required amount of chromatic aberration may be introduced without affecting the spherical corrections. The relation to be satisfied by components of powers $\mathrm{K}$ and $K^{\prime}$ made from glasses with $\nu$ values $\nu$ and $\nu^{\prime}$ to make the power of the combination for the $F$ line exceed that for the $C$ line by one $n^{t h}$ part of the power for the $D$ line is

$$
-\frac{\mathrm{K}}{\mathrm{K}^{\prime}}=\frac{\nu\left(n-v^{\prime}\right)}{v^{\prime}(n-v)} \text {. }
$$

This shows that the glass chart must be so constructed that the $v$ of any glass is replaced by $\left(\frac{1}{v}-\frac{1}{n}\right)^{-1}$. Fig. 4 shows the glasses of Fig. 3 replotted for $n=-100$, corresponding to very heavy overcorrection of the chromatic aberration, instead of $n=\infty$, the value for normal colour correction. Such a chart is very easily plotted for any required value of $n$ by direct calculation. An alternative method is to use Fig. 2, where the ordinates are the logarithms of the reciprocals of the abscissæ, for which both a uniform and an inverse scale are provided. If an achromatic objective is required, the ordinates for Fig. 3 are read from the curve of Fig. 2, taking the lower scale reading corresponding to the $v$ of the glass. If the dispersive power from $F$ to $\mathrm{C}$ is to be $1 / n^{\text {th }}$ of the power for the $\mathrm{D}$ line, the abscissa.selected is on the upper (uniform) scale a distance $\frac{1}{n}$ to the left of the $v$ value given by the lower scale, and the corresponding ordinate is to be plotted on the glass chart. Thus Fig. 4 would be obtained by displacing the $v$ scale through the distance ${ }^{\circ} 01$ to the right, measured on the upper scale, and using the modified ordinates so found as the ordinates in Fig. 4.† In this way, Fig. 2 enables a glass chart to be constructed for any required degree of chromatic under or over correction.

In this discussion reference has been made to the indices and dispersions for the $\mathrm{C}, \mathrm{D}$, and $\mathrm{F}$ lines. This is done simply by way of illustration. Figs. 1 and 2 are quite independent of any such assumption, and in plotting the glass charts any desired interpretation can be given to the quantities $\nu$ and $v^{\prime}$. The glasses must be plotted for the refractive

* This may be verified by noting that $\mathrm{K}+\mathrm{K}^{\prime}=\mathbf{l}$ and $\mathrm{K} / \nu+\mathrm{K}^{\prime} / \nu^{\prime}=\frac{1}{\nu^{\prime}(n-\nu)}$ if $\mathrm{K}={ }_{n\left(\nu-\nu^{\prime}\right)}^{\nu\left(n-\nu^{\prime}\right)}$ and $\mathrm{K}^{\prime}=-\frac{\nu^{\prime}(n-\nu)}{n\left(\nu-\nu^{\prime}\right)}$.

$\dagger$ It will be noticed that Fig. 2 is not drawn on the same scale as Figs. 1, 3, and 4. In use the same scale should be employed. 
index corresponding to the line of the spectrum for which the spherical conditions are to be applied.

By the use of such charts much time can be saved in calculating simple cemented objectives, since it is possible to ascertain beforehand that the glasses selected will be the most suitable of those that are available. Apart from such a method, only previous experience will enable a computer to avoid making calculations with a number of pairs of glasses, and after all having no result to show for his labours owing to the impossibility of meeting the various conditions that have to be satisfied with the pairs he has selected. This appears to be the direction in which the computer has hitherto had least systematic guidance. If a manufacturer knows that a certain effect has been obtained with certain glasses which are not now available, he can at once find what new combinations to take to secure a very similar result by using the charts to find the value of $4 \mathrm{C}_{0}+2 \tilde{\omega}+1$ for the old combination, and then performing the reverse operation with the glasses that can be obtained, selecting the pair that comes nearest to the old value of this quantity. The proper curvatures for the surfaces of the new lens will then be found in the usual way.

Formula for the calculation of the fundamental quantities referred to above will be found in the Physical Society paper to which reference has already been made, or they may be derived from formulæ previously given to the Optical Society* by making $\mathrm{C}$ a minimum as $r$ is varied.

\section{DISCUSSION.}

Mr. S. D. Chalmers said he was interested to see how Mr. Smith had developed his work by using charts. He himself had made use of a somewhat similar method. Another defect in the list of glasses was the absence of Medium Barium Crowns having a lower value of $v$ than at present. The principal interest in the charts lay in the fact that they saved a great deal of analytical work. In some cases, however, the results would reguire to be examined in detail as correction terms might have to be introduced.

The Author, in reply to a remark by Mr. Beck, said the charts related to the removal of first order coma. The effect of small errors would not increase the number of glasses available when good correction for more than one aberration had to be obtained. The same charts could be used for any of the first order aberrations, such as distortion, curvature, etc.

With regard to Mr. Chalmers' remarks he would like to point out that the charts contained a series of lines which allowed a correction to be applied to the first approximation if necessary.

* Trans. Opt. Soc. 17 (1917), 160. 